\title{
Edad gestacional, vía de parto y relación con el día y hora de nacimiento en dos instituciones del sector privado de salud
} Gestational age, mode of delivery, and relation to the day and time of birth in two private health care facilities

\author{
Dr. Ramón Larcade ${ }^{a}$ Dra. Norma Rossato ${ }^{b}$ Dr. Christian Belleccia, \\ Mag. Ariel Fernándezc, Dra. Cecilia García y Dr. Néstor Vain ${ }^{a, b, c}$ \\ Colaboradores: Ivana Liz Ruano y Lucía Segovia
}

\section{RESUMEN}

Introducción. A nivel mundial, hay una tendencia ascendenteen nacimientos prematuros y cesáreas. El objetivo fue describir edad gestacional (EG), vía de parto, distribución en días y horarios, y relación entre la vía de parto y el momento del nacimiento en dos instituciones privadas.

Población y métodos. Estudio prospectivo, transversal, analítico.

Resultados. Se incluyeron los 1500 nacidos vivos entre 9-2017 y 8-2018 (1465 embarazadas). El 99,4 \% fueron embarazos controlados; el 66,8 \% terminaron por cesárea. La causa fue cesárea previa en el $36,4 \%$, falta de progresión y descenso en el 18,9\% y elección materna en el 9,2\%. El peso promedio al nacer fue de $3232 \mathrm{~g} \pm 561,1 \mathrm{~g} \mathrm{y}$ la mediana de EG, 39 semanas (rango 38-40) por fecha de última menstruación. El 88,2\% fueron nacidos de término $\mathrm{y}$, de los prematuros, el $76,1 \%$ fueron pretérminos tardíos. Los nacidos de término temprano tuvieron mayor índice de cesáreas $(\mathrm{p}<0,001)$. Los nacimientos por cesárea en días hábiles fueron 849/1201 (el 74,5\%) y, en

a. Servicio de Neonatología, Sanatorio de la Trinidad Ramos Mejía.

b. Servicio de Neonatología, Sanatorio de la Trinidad Palermo.

c. Fundación para la Salud Materno Infantil (FUNDASAMIN).

Correspondencia:

Dr. Ramón Larcade: ramonlarcade@gmail. com

Financiamiento:

Ninguno.

Conflicto de intereses:

Ninguno que declarar.

Recibido: 8-6-2020

Aceptado: 20-7-2020

Cómo citar: Larcade R, Rossato N, Bellecci C, Fernández A, et al. Edad gestacional, vía de parto y relación con el día y hora de nacimiento en dos instituciones del sector privado de salud. Arch Argent Pediatr 2021;119(1):18-24.

\section{INTRODUCCIÓN}

Los trastornos relacionados con la duración de la gestación y el crecimiento fetal están entre las causas principales de morbimortalidad neonatal. ${ }^{1,2}$ A nivel mundial, hay una tendencia ascendente de nacimientos prematuros. Cada año, nacen, aproximadamente, 15 millones de niños pretérmino y un millón muere por trastornos relacionados con la prematuridad, principal causa de mortalidad infantil y segunda causa en los menores de 5 años. ${ }^{3,4}$

En la Argentina, hubo 685394 recién nacidos (RN) vivos en 2018; el $8,8 \%$ fueron prematuros. Estos niños presentan mayor riesgo de complicaciones a corto y largo plazo, como los problemas respiratorios, la retinopatía del prematuro, los trastornos del neurodesarrollo y la parálisis cerebral.5,6

Los nacimientos por cesárea están en aumento a nivel mundial. La mayor morbilidad neonatal asociada a la cesárea se reduce en forma significativa cuando esta se realiza luego de las 39 semanas cumplidas de edad gestacional (EG). ${ }^{7-10} \mathrm{La}$ elección de la vía de parto podría estar relacionada con causas no médicas e influir en la morbimortalidad materna y neonatal. ${ }^{11}$

En la Argentina, en el subsector de instituciones privadas de salud, no se cuenta con datos publicados recientes sobre la vía de nacimiento, aunque se sabe, por comunicaciones personales, que la cesárea es la más frecuente..$^{9-12}$ Conocer la frecuencia actual y las 
principales causas de cesárea permitiría diseñar protocolos de atención adecuados ante su alto índice. ${ }^{13,14}$ El objetivo fue describir la EG, la vía de parto, la distribución en días y horarios, y la relación entre la vía de parto y el momento del nacimiento en dos instituciones privadas.

\section{POBLACIÓN Y MÉTODOS}

Estudio prospectivo, transversal, analítico. Participaron dos instituciones privadas del Área Metropolitana de Buenos Aires, que atendían a una población con cobertura médica prepaga en su mayoría y un promedio de 2000 partos anuales en ese momento. Considerando la representatividad de los nacimientos para cada día de la semana y la factibilidad del estudio, se decidió un tamaño muestral por conveniencia de 1500 RN entre ambas instituciones. Fueron elegibles todos los $\mathrm{RN}$ vivos en forma consecutiva con peso $\geq 500$ g durante el período de estudio.

La información fue recolectada en forma prospectiva de los registros médicos maternos y neonatales (historias clínicas, ecografía obstétrica). Se utilizó un formulario diseñado para tal fin y cargado en una base de datos electrónica, y se aseguró anonimizarla.

\section{Variables}

Del embarazo: edad de la madre, antecedentes obstétricos y patología durante el último embarazo. Se consideraron embarazos controlados aquellos con $\geq 5$ controles prenatales, a partir del primer trimestre.

Del RN: peso en gramos, EG en semanas, vía de nacimiento (vaginal o cesárea y la causa según la historia clínica obstétrica). La EG se estableció por tres métodos: fecha de última menstruación (FUM), EG registrada en la historia clínica materna por el equipo obstétrico y ecografía precoz (hasta las 20 semanas de gestación). Para el cálculo de resultados, se eligió, de contarse con el dato, la EG por FUM. ${ }^{15}$

Se consideró RN de término a aquellos con EG $37-41^{+6}$ semanas; RN de término temprano a los nacidos con EG $37-38^{+6}$ semanas y RN de término completo a los nacidos con 39-41 ${ }^{+6}$ semanas. Se consideró RN pretérmino a aquellos con EG $22-36^{+6}$ semanas y RN pretérmino tardío a los nacidos entre las 34 y las $36^{+6}$ semanas. ${ }^{16}$

Se registró el momento del nacimiento con fecha, día de la semana, horario de nacimiento y número de días faltantes para el siguiente feriado con la hipótesis de que podría ser un motivo para el aumento de los nacimientos por decisión obstétrica no relacionada con condiciones maternas o fetales.

\section{Aspectos éticos}

Este protocolo y el formulario de recolección de datos fueron aprobados por los Comités de Ética del Centro de Educación Médica e Investigaciones Clínicas "Norberto Quirno" (CEMIC) y la Universidad Austral, y los Comités de Docencia de ambas instituciones.

\section{Análisis estadístico}

Se utilizó el software de Stata ${ }^{\circledR}$ versión 11. Se aplicó estadística descriptiva; las variables continuas se describieron como media y desvío estándar (DE); las variables categóricas, mediante frecuencias y porcentajes.

Se empleó el test $t$ de Student para la comparación de las variables continuas y el test de $\mathrm{chi}^{2}$ para comparar las variables categóricas. Se consideró el valor de $\mathrm{p}<0,05$ como estadísticamente significativo. Se expresó la fuerza de la asociación mediante odds ratio $(O R)$ e intervalo de confianza (IC) del $95 \%$. Se efectuó un análisis multivariado de regresión logística para evaluar los factores asociados a la indicación de cesárea.

\section{RESULTADOS}

La recolección de datos se llevó a cabo en el Sanatorio de la Trinidad Palermo entre

TABla 1. Datos maternos (N: 1465)

\begin{tabular}{lc}
\hline Datos maternos & $\mathbf{N}(\%)$ \\
\hline Edad materna (años) media \pm DE & $32,7 \pm 5,25$ \\
Número de gestas & $622(42,5)$ \\
$\quad 1$ & $843(57,5)$ \\
$\quad 2$ & $1457(99,4)$ \\
Embarazo controlado & $1414 / 1465(96,5)$ \\
Ecografía precoz & $7,7 \pm 2,1$ \\
EG (semanas), media \pm DE & $1347 / 1465(91,9)$ \\
FUM conocida & $486 / 1465(33,2)$ \\
Vía de nacimiento & 484 \\
$\quad$ Vaginal & 2 \\
$\quad$ Con feto único & $979 / 1465(66,8)$ \\
$\quad$ Doble & 944 \\
$\quad$ Córea & $34(1$ feto muerto $)$ \\
$\quad$ Doble único & $1($ un solo RN incluido) \\
$\quad$ Triple & $454 / 1465(31,0)$ \\
Cesárea anterior & $375 / 1465(25,6)$ \\
1 cesárea previa & $79 / 1465(5,4)$ \\
$\geq 2$ &
\end{tabular}

DE: desvío estándar; FUM: fecha de la última menstruación; EG: edad gestacional; RN: recién nacido. 
septiembre de 2017 y enero de 2018, y en el Sanatorio de la Trinidad Ramos Mejía entre mayo y agosto de 2018. El momento en que se hizo la investigación en cada centro dependió de la disponibilidad de un investigador responsable para asegurar la continuidad en el registro.

Durante el período de estudio, ingresaron 1465 mujeres embarazadas; 1428 con feto único, 36 embarazos gemelares (en un caso, con un feto muerto) y 1 embarazo triple, en el que se incluyó solo el primer RN por haber alcanzado el tamaño muestral estimado. Los $1500 \mathrm{RN}$ vivos fueron incluidos en el estudio.

\section{Descripción de los embarazos}

Se analizaron 1465 embarazos (Tabla 1). En 144/1465 (el 9,8 \%), se efectuó la inducción del trabajo de parto; 86/144 (el 59,7\%) terminaron por cesárea y 58/144 (el 40,3\%), por parto vaginal.

Además de las indicaciones fetomaternas, entre las causas de cesárea, se destacaron la cesárea previa (n: 356/979; el 36,4\%), la falta de progresión y descenso (n: 185/979; el 18,9\%) y la elección materna (n: 90/979; el 9,2 \%). En la Tabla 2, se observan los factores asociados en forma independiente a la realización de una cesárea en el análisis multivariado.
Entre las 622 madres primigestas, en 407 (el 65,4 \%), se realizó operación cesárea. La causa referida fue la falta de progresión y descenso en $133($ el $32,7 \%)$ y la elección materna en 50 (el 12,3\%).

\section{Descripción de los recién nacidos}

El peso promedio de nacimiento fue de $3232 \mathrm{~g}$ (DE $\pm 561,1 \mathrm{~g})$. La mediana de EG fue de 38 semanas (rango intercuartilo-RIC-37-39) por ecografía precoz y de 39 semanas (RIC 38-40) por FUM.

\section{Recién nacidos de término y prematuros}

De los 1500 RN, 1324 (el 88,3 \%) fueron de término y 176 (el 11,7\%) fueron prematuros. Entre los RN de término, 592 (el 44,7\%) fueron RN de término temprano. En este grupo, fue más frecuente el nacimiento por cesárea (el 74,5 \% vs. el 59,4\%) y la cesárea por cesárea previa (el 32,4 \% vs. el $19,3 \%$ ) comparado con los de término completos.

De los RN pretérmino, 134 (el 76,1\%) fueron pretérminos tardíos. En el conjunto de los RN prematuros tardíos más los de término temprano (726), se observó una mayor frecuencia de nacimiento por cesárea (546) comparado con los

TABLA 2. Factores asociados a la realización de cesárea. Análisis multivariado

\begin{tabular}{lccc}
\hline Variables & Odds ratio & IC 95 \% & Valor de $p$ \\
\hline Cesárea previa & 12,7 & $8,46-19,08$ & 0,000 \\
Aborto previo & 1,58 & $1,1-2,27$ & 0,013 \\
Embarazo múltiple & 11,74 & $4,04-34,12$ & 0,000 \\
Macrosomía & 15,09 & $3,54-64,18$ & 0,000 \\
RCIU & 6 & $1,73-20,79$ & 0,005 \\
Oligoamnios-polihidramnios & 5,65 & $1,96-16,28$ & 0,001 \\
HTA inducida por el embarazo & 4,8 & $2,1-11,08$ & 0,000 \\
Horario diurno & 5,11 & $3,38-7,75$ & 0,000 \\
\hline
\end{tabular}

RCIU: restricción del crecimiento intrauterino; HTA: hipertensión arterial; IC: intervalo de confianza.

Tabla 3. Distribución de los recién nacidos según el día de la semana y su relación con los feriados

\begin{tabular}{|c|c|c|c|}
\hline Día de la semana & $\begin{array}{l}\text { Recién nacidos } \\
\text { N }(\%)\end{array}$ & Vía vaginal & Cesárea \\
\hline De lunes a jueves no víspera de feriados (128 días) & $\begin{array}{l}916(61,1) \\
7,1 / \text { día }\end{array}$ & $271(55,53)$ & $645(63,74)$ \\
\hline Viernes y víspera de feriado (37 días) & $\begin{array}{c}285(19,0) \\
7,7 / \text { día }\end{array}$ & $91(18,64)$ & $194(19,17)$ \\
\hline Sábado-domingo-feriado (80 días) & $\begin{array}{c}299(19,9) \\
\text { 3,7/ día }\end{array}$ & $126(25,82)$ & $173(17,09)$ \\
\hline Total & $1500(100,0)$ & $488(100,00)$ & $1012(100,00)$ \\
\hline
\end{tabular}


RN de $\geq 39$ semanas (435) (el 75,2\% vs. el 59,4\%, OR 2,1; 1,64-2,6; $\mathrm{p}<0,001)$.

\section{Día y vía de nacimiento}

El período de estudio abarcó 245 días. Nacieron 1201 niños en días de semana no feriados (7,3/ día) y 299 niños durante sábadodomingo-feriado (3,7/ día) (Tabla 3). El índice de cesáreas en días de semana laborables fue del $69,85 \%$ y, sábado-domingo-feriados, el 57,85\% (OR 1,67; 1,27-2,18; p < 0,001) (Figura 1).

\section{Horario de nacimiento y vía de parto}

Desde las 22:00 hasta las 8:00, predominaron los partos vaginales (174/289; un 60,2\%); a partir de las 08:00, se incrementaron los nacimientos por cesárea, que persistieron elevados hasta las 22:00 (897/1211; un 74,1 \% de cesáreas) (Figura 2). Tomando como referencia el horario de 00:00 a 05:00, hubo una relación estadísticamente significativa entre el horario de nacimiento y la realización de cesárea como vía de parto (Tabla 4).

FiguRa 1. Número de recién nacidos según el día de la semana y la vía de parto

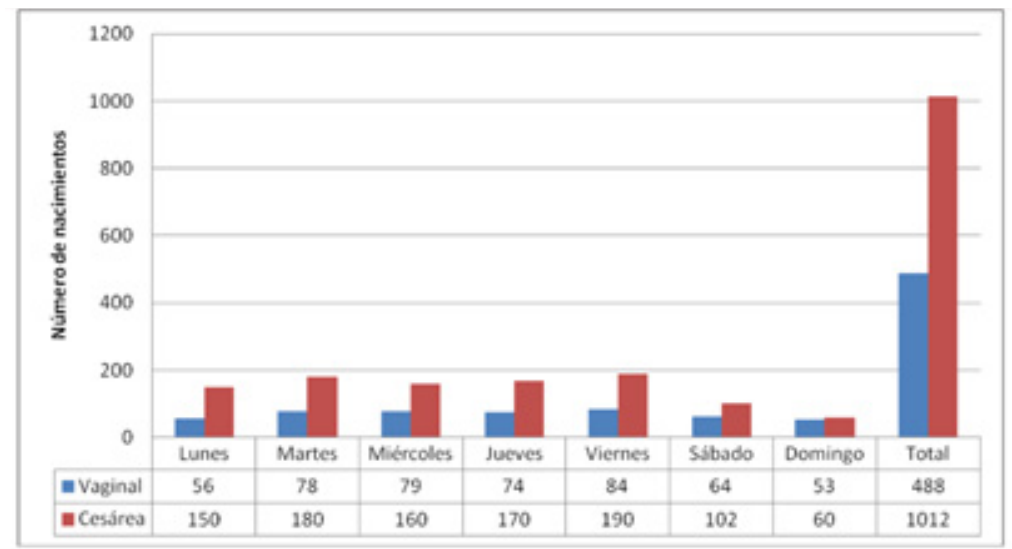

FIGURA 2. Horario de nacimiento y vía de parto de los recién nacidos

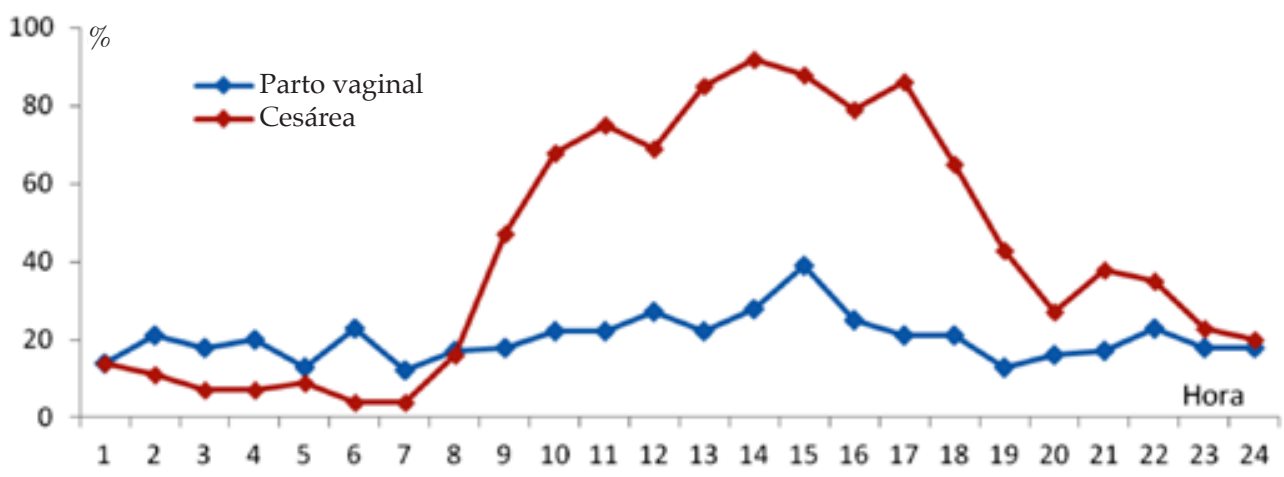

TABLA 4. Relación entre horario y vía de nacimiento

\begin{tabular}{lcccc}
\hline Franja horaria & Nacimientos & Vía vaginal & Cesárea & Nacimiento por cesárea OR (IC 95 \%) \\
\hline 00:00-05:59 & 161 & $\mathbf{1 0 9}(67,7)$ & $\mathbf{5 2}(\mathbf{3 2 , 3})$ & $\mathbf{1}$ \\
06:00-11:59 & 397 & $118(29,72)$ & $279(70,28)$ & $4,96(3,34-7,35)$ \\
$\mathbf{1 2 : 0 0 - 1 7 : 5 9}$ & $\mathbf{6 5 1}$ & $\mathbf{1 5 6}(23,96)$ & $\mathbf{4 9 5}(\mathbf{7 6 , 0 4 )}$ & $\mathbf{6 , 6}(\mathbf{4 , 5 7 - 9 , 7 1 )}$ \\
18:00-23:59 & 291 & $105(36,08)$ & $186(63,92)$ & $3,7(2,47-5,61)$ \\
\hline
\end{tabular}

OR: odds ratio; IC: intervalo de confianza. 


\section{DISCUSIÓN}

Durante el período de estudio, el $66,8 \%$ de los nacimientos ocurrió por cesárea y, entre las principales causas, estuvieron la cesárea previa (el $36,4 \%$ ), la falta de progresión y descenso (el 18,9\%) y la elección materna (el 9,2\%). La proporción de nacimientos por cesárea ha ido en aumento en los últimos 35 años, sobre todo, de aquellas que no pueden ser adjudicadas a complicaciones o riesgos maternos ni fetales. ${ }^{19}$

En América Latina y el Caribe, el promedio de la tasa de cesáreas ronda el $42 \%$ y llega, en algunos países, al $70 \%$ o superiores. ${ }^{17}$ Incluso en algunas instituciones privadas de salud de Brasil, la tasa de cesáreas es cercana al $90 \% .{ }^{18}$ En la Argentina, la tasa de cesáreas también ha ido en aumento en los últimos años. ${ }^{19}$ Dos instituciones privadas de Buenos Aires comunicaron que, entre 2004 y 2007, la frecuencia de cesáreas en RN de término fue cercana al $50 \% .^{9,12}$ Esa frecuencia y la de nuestra cohorte exceden ampliamente la comunicada para el medio público. ${ }^{19}$

No se cuenta, en nuestro país, con información precisa ni reciente acerca de la proporción de nacimientos por cesárea sobre el total de los que ocurren en el medio privado. Las instituciones de salud deberían llevar estadísticas basadas en el Sistema de Información Perinatal (SIP) u otra base de datos y sería deseable una norma de salud pública que obligara a la comunicación de estos datos. ${ }^{20}$ Muchos factores culturales, sociales y del sistema de atención pueden influir en la diferencia de incidencia de cesáreas entre el medio privado y el público, como la exigencia de atención personalizada por parte de las pacientes, las bajas remuneraciones y la litigiosidad en obstetricia, entre muchas otras.

Los diagnósticos registrados como motivo del nacimiento por cesárea en nuestro estudio coinciden parcialmente con los publicados por Barber y cols., donde las principales causas de cesárea primaria fueron falta de progresión y descenso, alteración de la vitalidad fetal y presentación fetal anómala, entre otras. ${ }^{21}$ Las indicaciones de cesárea comunicadas en nuestra cohorte difieren de las sugeridas por la Organización Mundial de la Salud (OMS).22

En la actualidad, es muy poco frecuente el parto vaginal luego de una cesárea. ${ }^{14,23} \mathrm{La}$ alta tasa de cesárea en nulíparas, posiblemente, lleve a que, en estas madres, el número de cesáreas aumente. La indicación de cesárea por el antecedente de una cesárea anterior es controvertida. Se debería informar a la embarazada sobre los potenciales beneficios de un parto vaginal siempre y cuando las condiciones materno-fetales lo permitan. ${ }^{24}$

En nuestro trabajo, se registró, en la muestra general, un 9,2 \% de cesáreas por elección materna (un 12,3\% en el grupo de nulíparas). Respecto a esta forma de terminación del embarazo, en los últimos años, la comunidad médica ha sido testigo de un cambio social y cultural relacionado con el empoderamiento de las mujeres en diferentes áreas y aspectos, desde la elección del momento oportuno para transitar su maternidad a la elección del método reproductivo y el número de hijos que desean tener.

Es muy importante, para que la toma de decisiones sea de manera informada, que la mujer embarazada y su pareja conozcan y comprendan los riesgos y beneficios en la elección de esta vía de nacimiento cuando transitan embarazos de bajo riesgo tanto en términos del impacto potencial en la madre como en el RN y la relación con el momento elegido para la intervención. En este aspecto fundamental, el equipo asistencial debe estar comprometido. ${ }^{17}$

Se encontró una diferencia estadísticamente significativa en el índice de cesáreas en los nacidos a término temprano comparados con los de término completo. Esto coincide con lo observado en los últimos años, junto con un incremento de los partos inducidos y los nacimientos por cesárea. Diferentes estudios muestran que la población de $\mathrm{RN}$ a término temprano presenta mayor morbilidad neonatal comparada con los nacidos entre las 39 y las 41 semanas. $^{25-28}$

Estos resultados coinciden con los de Martínez-Nadal y col., que observaron una tasa de cesáreas significativamente superior en los $\mathrm{RN}$ de término temprano y una mayor proporción de ingresos a la Unidad de Cuidados Intensivos Neonatales y morbilidad en general comparados con los RN de 39-41 semanas. ${ }^{28}$ Hourani y cols., encontraron mayor morbilidad respiratoria, hipotermia y dificultad en la alimentación en RN de término temprano comparados con los nacidos de 39 semanas o más. ${ }^{11}$

A nivel mundial, hay una tendencia en aumento de los partos prematuros. ${ }^{7,8,29,30}$ En nuestro estudio, el 11,7\% de los RN fueron pretérmino. Estas cifras superan los datos nacionales (el 8,8\% de nacidos pretérmino), derivados, primariamente, de registros de hospitales públicos. ${ }^{5}$ La no obligatoriedad de su comunicación y la falta de formato de registro unificado para el subsector privado generan una 
ausencia de datos confiables. Nuestro trabajo no contempló la evaluación de la morbilidad neonatal, dado que era una muestra pequeña para obtener conclusiones válidas. Sin embargo, es bien conocida la mayor morbilidad asociada a la prematuridad. ${ }^{8}$

Los RN pretérminos tardíos, en nuestro estudio, representaron el $76 \%$ de los nacidos pretérmino. Estos datos coincidieron con las publicaciones internacionales. ${ }^{6,30}$ La importancia de conocer su incidencia radica en "visibilizarlos", ya que, muchas veces, este grupo es subestimado desde el punto de vista del cuidado asistencial por ser erróneamente considerados como RN "casi" a término. Estos niños presentan mayor mortalidad y morbilidades asociadas, como dificultad respiratoria, hipoglucemia, trastornos en la termorregulación, en la succión-deglución, hiperbilirrubinemia, mayor susceptibilidad a infecciones y reinternaciones. A largo plazo, tienen también peor desempeño escolar (lectoescritura y matemáticas). ${ }^{30-34}$

De lunes a viernes, hubo entre dos y tres veces más nacimientos por cesárea que por vía vaginal. Los domingos, en cambio, la frecuencia de nacimientos fue menor y la cantidad de nacimientos por parto vaginal y cesárea fue muy similar. Es probable que esta diferencia esté vinculada con la programación de los nacimientos con cesáreas electivas en días hábiles por conveniencia de la familia o del equipo obstétrico.

Estos resultados coinciden con los de Del Carmen y cols., que evaluaron la relación entre el día de la semana y la vía de nacimiento. Estos autores encontraron que las madres tenían entre el $25 \%$ y el $29 \%$ menos de probabilidad de tener una cesárea en los días de fin de semana o feriado comparados con los días de semana. ${ }^{35}$ En la misma línea, Young y cols. describieron un fenómeno denominado "efecto del fin de semana", momento en el que se producían menos nacimientos y menor porcentaje de cesáreas comparados con los días de semana. ${ }^{36}$

Respecto a la hora de nacimiento, se pudo observar que los nacimientos aumentaron de modo progresivo desde las $7 \mathrm{~h}$ hasta las $14 \mathrm{~h}$ y persistieron elevados hasta las $21 \mathrm{~h}$. Coincidentemente, las cesáreas, a partir de las $8 \mathrm{~h}$, también se incrementaron y persistieron elevadas hasta las $22 \mathrm{~h}$. Fue muy notable que, entre las 9 y las $18 \mathrm{~h}$, las cesáreas duplicaron y, por momentos, triplicaron el número de partos vaginales.

Nam y cols. y Pasupathy y cols., entre otros, describieron, en estudios observacionales, un peor pronóstico en las madres y los $\mathrm{RN}$ asistidos en horarios semanales nocturnos o de fines de semana y feriados, comparados con los diurnos o en días laborales. Brookfield y cols., en cambio, no encontraron una asociación significativa en la morbilidad neonatal grave entre los grupos evaluados (nacidos en horarios diurno, nocturno o madrugada). ${ }^{37-39}$

En nuestro trabajo, cuando se analizó el momento del nacimiento y su cercanía a los fines de semana o feriados, se observó que el $19 \%$ de los nacimientos se produjeron los viernes o en vísperas de feriado próximo. Esto coincidió con lo comunicado por Mola y cols., respecto a una mayor frecuencia de nacimientos e ingresos de $\mathrm{RN}$ pretérminos tardíos a la Unidad de Neonatología los viernes. Estos autores lo denominaron "el efecto de viernes". ${ }^{40}$

La información obtenida en este estudio sugiere que los nacimientos ocurrieron con una cronología de días y horarios, posiblemente, más relacionada con las decisiones del equipo médico y/o de la familia que con causas vinculadas a la salud materna o fetal. Por múltiples referencias personales, se sabe que esta situación es común a muchas instituciones privadas de nuestro país.

Estos datos podrían ser de utilidad a cada centro que enfrente una problemática similar para organizar la distribución del recurso humano y la optimización del área de nacimientos e internaciones. Asimismo, sería beneficiosa la implementación de la obligatoriedad de proveer oficialmente registros de estas características desde todos los centros privados donde se producen nacimientos con el objetivo de diseñar estrategias de cuidado o políticas de salud que enfrenten el problema del alto índice de cesáreas.

\section{CONCLUSIONES}

La mediana de EG fue de 39 semanas. La cesárea fue la vía de nacimiento más frecuente. Los nacimientos se produjeron, predominantemente, de lunes a viernes entre las 8 y las $21 \mathrm{~h}$. El 82,9\% de los nacimientos por cesárea ocurrieron en días de semana laborables.

\section{REFERENCIAS}

1. Finkelstein JZ, Duhau M, Fasola ML, Escobar P. Mortalidad neonatal en Argentina. Análisis de situación de 2005 a 2014. Arch Argent Pediatr. 2017; 115(4):343-9.

2. Lona Reyes JC, Pérez RamírezRO, Llamas Ramos L, Gómez Ruiz LM, et al. Mortalidad neonatal y factores asociados en recién nacidos internados en una Unidad de Cuidados Neonatales. Arch Argent Pediatr. 2018; 116(1):42-8.

3. Howson CP, Kinney MV, Lawn JE (eds.). Born Too Soon: The Global Action Report on Preterm Birth. Geneva: March 
of Dimes, PMNCH, Save the Children, WHO; 2012.

4. Beck S, Wojdyla D, Say L, Betran AP, et al. The worldwide incidence of preterm birth: a systematic review of maternal mortality and morbidity. Bull World Health Organ. 2010; 88(1):31-8

5. Dirección de estadísticas e información en salud. Indicadores básicos. Argentina. 2018. [Acceso: 20 de abril de 2020]. Disponible en: http:/ / www.deis.msal.gov.ar/ wp-content/uploads / 2019/02/Indicadores-Basicos-2018. pdf.

6. Delnord M, Zeitlin J. Epidemiology of late preterm and early term births - An international perspective. Semin Fetal Neonatal Med. 2019; 24(1):3-10.

7. Spong CY, Mercer BM, D'alton M, Kilpatrick S, et al. Timing of indicated late-preterm and early-term birth. Obstet Gynecol. 2011; 118(2 Pt 1):323-33.

8. PurischSE, Gyamfi-Bannerman C. Epidemiology of preterm birth. Semin Perinatol. 2017; 41(7):387-91.

9. Ceriani Cernadas JM, Mariani G, Pardo A, Aguirre A, et al. Nacimiento por cesárea al término en embarazos de bajo riesgo: efectos sobre la morbilidad neonatal. Arch Argent Pediatr. 2010; 108(1):17-23.

10. Reddy UM, Ko CW, Raju TNK, Willinger M. Delivery indications at late-preterm gestations and infant mortality rates in the United States. Pediatrics. 2009; 124(1):234-40.

11. Hourani M, ZiadeF, Rajab M. Timing of planned caesarean section and the morbidities of the newborn. $N \mathrm{Am} \mathrm{J} \mathrm{Med}$ Sci. 2011; 3(10):465-8.

12. Armadans M, Osorio MF, Pedicone $C$, Durán $P$, et al. Morbilidad en recién nacidos de término en relación a su edad gestacional. Rev Chil Pediatr. 2010; 81(5):402-8.

13. Zanardo V, Simbi AK, Franzoi M, Soldà G, et al. Neonatal respiratory morbidity risk and mode of delivery at term: influence of timing of elective caesarean delivery. Acta Paediatr. 2004; 93(5):643-7.

14. American College of Obstetricians and Gynecologists; Society for Maternal-Fetal Medicine; Caughey AB, Cahill AG, etal. Safe Prevention of the Primary Cesarean Delivery. Am J Obstet Gynecol. 2014; 210(3):179-93.

15. Macaulay S, Buchmann EJ, Dunger DB, Norris SA. Reliability and validity of last menstrual period for gestational age estimation in a low-to-middle-income setting. J Obstet Gynaecol Res. 2019; 45(1):217-25.

16. Gill JV, Boyle EM. Outcomes of infants born near term. Arch Dis Child. 2017; 102(2):194-8.

17. Mariani GL, Vain NE. The rising incidence and impact of non-medically indicated pre-labour cesarean section in Latin America. Semin Fetal Neonatal Med. 2019; 24(1):11-7.

18. Agência Nacional de Saúde Suplementar. Painel de indicadores de atenção materna e neonatal. [Acceso: 28 de junio de 2020]. Disponible en: http:/ / www.ans.gov.br/ perfil-do-setor/dados-e-indicadores-do-setor/ painel-deindicadores-da-atencao-materna-e-neonatal.

19. Ministerio deSalud y DesarrolloSocial.Gráfico 9.Evolución de la Tasa de cesárea, porcentajes e intervalos de confianza del 95\%. Total, país. República Argentina. Años 2010-2018. En Sistema Informático Perinatal para la gestión (SIP-G). Indicadores básicos 2018. República Argentina. Buenos Aires: MINSAL; 2019:31. [Acceso: 28 de junio de 2020]. Disponibleen:http:/ / www.msal.gob.ar/images/stories/ bes / graficos / $0000001616 \mathrm{cnt}$-anuario-sip-2018.pdf.

20. Organización Panamericana de la Salud. Sistema de Información Perinatal. [Acceso: 3 de julio de 2020]. Disponible en: https://www.paho.org/clap/index. php?option $=$ com_content\&view $=$ article\&id $=84$ :sistemainformatico-perinatal\&Itemid=242\&lang $=$ en .
21. Barber EL, Lundsberg L, Belanger K, Pettker CM, et al. Contributing indications to the rising cesarean delivery rate. Obstet Gynecol. 2011; 118(1):29-38.

22. Organización Mundial de la Salud. Solo se deben practicar las cesáreas que sean necesarias por motivos médicos. Comunicado de prensa. Abril 2015. [Acceso: 3 de julio de 2020].Disponibleen:https:/ / www.who.int/mediacentre / news / releases / 2015/caesarean-sections/es/.

23. Ceriani Cernadas JM. La epidemia de cesáreas no justificadas, ¿podremos revertirla? Arch Argent Pediatr. 2019; 117(2):66-7.

24. Sociedad de Obstetricia y Ginecología de Buenos Aires. Parto vaginal después de una cesárea. 2019. [Acceso: 3 de julio de 2020]. Disponibleen: http:/ / sogiba.org.ar/images/ Parto_Vaginal_despues_de_una_Cesarea_SOGIBA\%20 2019.pdf.

25. Fang YMV, Guirguis P, Borgida A, Feldman D, et al. Increased neonatal morbidity despite pulmonary maturity for deliveries occurring before 39 weeks. J Matern Fetal Neonatal Med. 2013; 26(1):79 $\square 82$.

26. Bates E, Rouse DJ, Mann ML, Chapman V, et al. Neonatal outcomes after demonstrated fetal lung maturity before 39 weeks of gestation. Obstet Gynecol. 2010; 116(6):1288ロ 95 .

27. Ceriani Cernadas JM. Los cambios en la definición del recién nacido de término y su implicancia en los cuidados perinatales. ¿Se están cumpliendo? Arch Argent Pediatr. 2017; 115(5):410-1.

28. Martínez-Nadal S, Demestre X, Raspall F, Álvarez JA, et al. Morbilidad neonatal en los recién nacidos a término precoz. An Pediatr (Barc). 2014; 81(1):39 $\square 44$.

29. Goldenberg RL, Culhane JF, Iams JD, Romero R. Epidemiology and causes of preterm birth. Lancet. 2008; 371(9606):75-84.

30. Stewart DL, Barfield WD, COMMITTEE ON FETUS AND NEWBORN. Updates on an At-Risk Population: Late-Preterm and Early-Term Infants. Pediatrics. 2019; 144(5):e20192760.

31. Kugelman A, Colin AA. Late Preterm Infants: Near Term ButStill in a Critical Developmental Time Period. Pediatrics. 2013; 132(4):741-51.

32. Machado LCJr, Passini RJr, Rosa IR, CarvalhoHB. Neonatal outcomes of late preterm and early term birth. Eur J Obstet Gynecol Reprod Biol. 2014; 179:204ロ8.

33. Pulver LS, Guest-Warnick G, Stoddard GJ, Byington CL, et al. Weight for gestational age affects the mortality of late preterm infants. Pediatrics. 2009; 123(6):e1072-7.

34. Wang ML, Dorer DJ, Fleming MP, Catlin EA. Clinical outcomes of near-term infants. Pediatrics. 2004;114(2):372-6.

35. Del Carmen GA, Stapleton S, Qadan M, Del Carmen MG, et al. Does the Day of the Week Predict a Cesarean Section? A Statewide Analysis. J Surg Res. 2020; 245:288-94.

36. Young D. When was the baby born? Reflecting on the time of day, day of week, mode of delivery, and birth outcomes. Birth. 2011; 38(1):1-2.

37. Nam JY, Lee SG, Nam CM, Park S, et al. The effect of offhour delivery on severe maternal morbidity: a populationbased cohort study. Eur J Public Health. 2019; 29(6):1031-6.

38. Pasupathy D, Wood AM, Pell JP, Fleming M, et al. Time of birth and risk of neonatal death at term: retrospective cohort study. BMJ. 2010; 341:c3498.

39. Brookfield KF, O'Malley K, El-Sayed YY, Blumenfeld YJ, et al. Does Time of Delivery Influence the Risk of Neonatal Morbidity? Am J Perinatol. 2016; 33(5):502-9.

40. MolaS, Graeber JE, Polak MJ, Yossuck P. Is friday the busiest for late preterm delivery? W V Med J. 2010; 106(5):10-3. 